\title{
ROBUST CREDIBILITY VIA ROBUST KALMAN FILTERING
}

\author{
By ERHARd Kremer
}

Hamburg

\begin{abstract}
Credibility theory is closely related to Kalman filtering. As a consequence, methods proposed for robustifying the Kalman filter can often be specialised to obtain robust credibility rating procedures. The application of one such method to several classical credibility models is shown in this paper.
\end{abstract}

\section{INTRODUCTION}

Credibility theory is a very old branch of risk theory and non-life insurance mathematics. Eearly results are by MOWBRAY (1914) and WhITNEY (1918). A theoretically elegant approach was given by BüHLMANN (1967) and BÜHLMANN \& Straub (1970).

The classical models presented by those authors can be generalised to regression models, hierarchical models and evolutionary models. Generalisations have been studies intensively in the actuarial literature over the past twenty years. Some key references are HACHEMEISTER (1975), TAYLOR (1979), SUNDT (1980, 1983), NORBERG (1980, 1986), KREMER (1988a, 1988b).

In later years several authors have investigated ways of robustifying credibility rating methods. The aim of robustification is to limit the influence of extremely large claim amounts on the estimated premium. The reader is referred to, e.g. Gisler (1980), Bülmainn et al. (1982), Kremer (1991), KünSCH (1992), GisLer $\&$ REINHARD (1993).

MEHRA (1973) pointed out that credibility estimation can be achieved by the Kalman filtering technique. DE Jong \& ZEHNWIRTH (1983) explored the correspondence for the classical credibility models, and ZEHNwIRTH (1985) explored its implications for evolutionary models.

Robust versions of the Kalman filter have been studies for some time, for example by Masreliez \& MarTin (1977), Meinhold \& Singpurwalla (1989) and CIPRA \& ROMERA (1991). Due to the close relation between Kalman filtering and credibility theory, it is obvious that corresponding robust versions of credibility rating techniques can be derived. In the present paper we specialise the method proposed by CIPRA \& ROMERA (1991) to the three most important credibility models. The resulting robust credibility techniques turn out to be quite tractable. 


\section{PRELIMINARIES}

Suppose that all probabilistic statements are based on a probability space $(\Omega, \Delta, P)$ and consider a risk during periods with indices $i=1,2,3, \ldots, n+l$. Assume that the claims behavior of a risk over all periods can be described by a parameter $\theta$. Suppose that the value of this parameter is unknown and interpret it as a realisation of a random variable:

$$
\theta:(\Omega, \Delta, P) \rightarrow(\Theta, \tau),
$$

with the parameter space $\Theta$ and the $\sigma$-algebra $\tau$ on $\Theta$. Let the observed claims amounts (or loss ratios) of the risk be represented by the nonnegative random variables:

$$
X_{i}, \quad \text { with } \quad i=1,2,3, \ldots, n+1,
$$

defined on $(\Omega, \Delta, P)$. It is assumed that all $X_{i}$ lie in the Hilbert space $L_{2}$ of measurables, square integrable functions $f$ (identified with the equivalence class of all $g$ which are $P$-a.e. equal to $f$ ) defined on $\Delta$ with scalar product :

$$
\left\langle f_{1}, f_{2}\right\rangle=E\left(f_{1} \cdot f_{2}\right)
$$

and norm :

$$
\|f\|=E\left(f^{2}\right)^{1 / 2} .
$$

In the given insurance context the conditional expectation

$$
m_{i}=E\left(X_{i} \mid \theta\right)
$$

is called the net premium (or net loss ratio) in period no. $i$. Then the credibility estimator is nothing else but the linear-affine prediction of $m_{n+1}$ from $X_{1}, X_{2}, \ldots, X_{n}$. Defining the subspace $A_{n}$ of all linear-affine combinations

$$
f_{n}=a_{0}+\sum_{i=1}^{n} a_{i} \cdot X_{i},
$$

the credibility estimator is defined as the projection of $m_{n+1}$ on $A_{n}$, i.e. as the random variable $m_{n+1} \in A_{n}$ with

$$
\left\|m_{n+i}-\hat{m}_{n+1}\right\| \leq\left\|m_{n+1}-f_{n}\right\|
$$

for all $f_{n} \in A_{n}$.

In general the credibility estimator can be determined by solving certain normal equations. Under more special model assumptions one can derive explicit formulas for the credibility estimator. Three special models are given in the Section 4. Especially in the regression model one can calculate explicit credibility estimators, see e.g. NORBERG (1980).

It is well-known that the usual credibility estimators are not robust against extremely large claim amounts. This has led to attempts to contruct robust versions of the classical credibility estimators. One method to robustify the credibility estimator was given by Gisler already in 1980 . 
Alternative robustifications, which also cover the general regression model, are given by KREMER (1991).

\section{ROBUST KALMAN FILTERING}

The Kalman filter is a well-known instrument for recursive prediction in dynamic linear systems. A dynamic linear system is defined by the two stochastic linear recursions :

$$
\begin{aligned}
& X_{i}=H_{i} \cdot b_{1}+v_{i} \\
& b_{i}=F_{1} \cdot b_{i-1}+w_{i},
\end{aligned}
$$

where $X_{i}$ is a $p$-dimensional stochastic vector of observations, $H_{i}$ a known $(p x q)$-dimensional design matrix, $b_{i}$ a $q$-dimensional stochastic parameter vector and $F_{i}$ a known $(q x q)$-dimensional transition matrix. The $\nu_{i}, w_{i}$ are random disturbances with:

$$
\begin{array}{ll}
E\left(v_{i}\right)=0, & E\left(w_{i}\right)=0, \\
E\left(v_{i} \cdot v_{j}^{T}\right)=0, & E\left(w_{i} \cdot w_{j}^{T}\right)=0, \\
E\left(v_{i} \cdot v_{i}^{T}\right)=R_{i}, & E\left(w_{i} \cdot w_{i}^{T}\right)=Q_{i} \\
E\left(v_{i} \cdot w_{j}^{T}\right)=0, & i \neq j,
\end{array}
$$

where $R_{i}, Q_{i}$ are known covariance matrices. The Kalman filter algorithm gives handy recursions for the optimal affine-linear predictor of $b_{i}$ from $X_{1}, X_{2}, \ldots, X_{i-1}$. For more details see Section 3 in De Jong et al. (1983).

Like other standard methods the Kalman filter is not robust to outliers. As a consequence, several authors have proposed robust versions of the usual Kalman filter algorithm. Recently, a handy robutistification was proposed by CIPRA \& ROMERA (1991). We give a brief summary of their result.

Denote by $\hat{b}_{i}$, the one-step ahead prediction of $b_{i}$, based on the observations $X_{1}, \ldots, X_{i-1}$, and define its error covariance matrix as

$$
C_{i}=E\left(b_{i}-\hat{b}_{i}\right)\left(b_{i}-\hat{b}_{i}\right)^{T} \text {. }
$$

For a given covariance matrix $M$, we define as $M^{-1 / 2}$ any matric which satisfies $M^{-1 / 2} \cdot M \cdot\left(M^{-1 / 2}\right)^{T}=I$.

With this convention we now introduce the matrices

$$
\begin{aligned}
& A_{i}^{q \times q}=C_{i}^{-1 / 2}, \\
& D_{i}^{p \times q}=R_{i}^{-1 / 2} \cdot H_{i},
\end{aligned}
$$

and the random vectors

$$
\begin{aligned}
& s_{i}^{p \times 1}=R_{i}^{-1 / 2} \cdot X_{i}, \\
& p_{i}^{q \times 1}=A_{i} \cdot \hat{b}_{i} .
\end{aligned}
$$


Note that, conditional on $b_{i}$, the stochastic vector $s_{i}$ has mean $D_{i} \cdot b_{i}$ and covariance matrix $l$. Further note that

$$
E\left(p_{i}-A_{i} \cdot b_{i}\right)\left(p_{i}-A_{i} \cdot b_{i}\right)^{T}=I \text {. }
$$

In the spirit of $\boldsymbol{M}$-Estimation, Cipra \& ROMERA (1991) propose to determine the updated estimate $\hat{b}_{i}^{i}$ of $b_{i}$, given all observations $X_{1}, \ldots, X_{i}$, as the solution of a minimisation problem, namely

$$
\operatorname{minimise}\left\{\sum_{k=1}^{q} \rho_{1 k}\left(\rho_{k i}-a_{k i}^{T} \cdot \hat{b}_{i}^{i}\right)+\sum_{j=1}^{N} \rho_{2 j}\left(s_{j i}-d_{j i}^{T} \cdot \hat{b}_{i}^{i}\right)\right\}
$$

Here $\rho_{11}, \ldots, \rho_{1 q} \geq 0$ and $\rho_{21}, \ldots, \rho_{2 q} \geq 0$ are arbitrary robustifying functions, the $k$-th row of $A_{i}$ is denoted by $a_{k i}^{T}$, and the $j$-th row of $D_{1}$ is denoted by $d_{\mu l}^{T}$. Denote the derivatives of the functions $\rho$ by $\Psi$.

It is seen that the resulting estimator $\hat{b}_{i}^{i}$ will be the result of a compromise between the desire to minimise deviation from the one-step ahead prediction (note $p_{i}-A_{i} \cdot \hat{b}_{i}=0$ ) and the desire to have $\hat{b}_{i}^{i}$ reflect the information in the new data as represented by $s_{i}$.

Having solved (3.1) to obtain $\hat{b}_{i}^{i}$, one obtains the one-step ahead prediction to use in the next recursion by

$$
\hat{b}_{i+1}=F_{i+1} \cdot \hat{b}_{i}^{i}
$$

For $\hat{b}_{1}^{i}$ one has the normal equations (see (2.8) in CIPRA \& ROMERA (1991)):

$$
\sum_{k=1}^{q} a_{k i}^{T} \cdot \Psi_{1 k}\left(p_{k i}-a_{k i}^{T} \cdot \hat{b}_{i}^{i}\right)+\sum_{j=1}^{p} d_{j i}^{T} \cdot \psi_{2 j}\left(s_{j i}-d_{j r}^{T} \cdot \hat{b}_{i}^{i}\right)=0 .
$$

By approximating $\hat{b}_{i}^{i}$ by $\hat{b}_{i}$ (3.2) gives a certain approximate normal equation

$$
\sum_{k=1}^{q} w_{1 k i} \cdot a_{k l}^{T} \cdot\left(p_{k i}-a_{k i}^{T} \cdot \hat{b}_{i}^{i}\right)+\sum_{j=1}^{p} w_{2 j i} \cdot d_{j i}^{T} \cdot\left(s_{j i}-d_{j i}^{T} \cdot \hat{b}_{i}^{i}\right)=0
$$

where the weights $w_{1 k i}, w_{2 j i}$ are defined as:

$$
\begin{aligned}
& w_{1 k i}=\lim _{x \rightarrow 0}\left(\frac{\Psi_{1 k}(x)}{x}\right) \\
& w_{2 j i}=\frac{\Psi_{2 j}\left(s_{j i}-d_{j i}^{T} \cdot \hat{b}_{i}\right)}{s_{j i}-d_{j i}^{T} \cdot \hat{b}_{i}} .
\end{aligned}
$$

So far for the general robustification of the Kalman-filter. Turn now to more practicable, special cases.

It is reasonable to assume that the disturbances $w_{1}$ do not produce outliers. This results in the choice:

$$
\Psi_{1 k}(x)=x
$$


Furthermore one is willing to take:

$$
\Psi_{2 j}(\cdot)=\Psi(\cdot)
$$

independent of $j$, e.g. :

$$
\Psi_{2 j}(\cdot)=\Psi_{H}(\cdot)
$$

In the practical case $p=1$ one can choose the so called (one-sided) Huberfunction :

$$
\begin{aligned}
& \Psi_{H}(z)=z, \quad \text { for } \quad z \leq c \\
& =c, \quad \text { for } \quad z>c,
\end{aligned}
$$

where $c$ is a given positive constant. CIPRA \& ROMERA (1991) propose to take (3.2) in case of the Huber-function $\Psi_{H}$ for $\psi$ and the approximation (3.3) in case of general $\Psi$. For general $\psi$ the formula (3.3) gives the following recursion in case that $p=1$ :

$$
\hat{b}_{i}^{i}=\hat{b}_{t}+\left(\frac{C_{i} \cdot H_{i}^{T}}{H_{i} \cdot C_{i} \cdot H_{i}^{T}+R_{i} / k_{i}}\right) \cdot\left(X_{i}-H_{i} \cdot \hat{b}_{i}\right)
$$

where :

$$
\begin{gathered}
C_{i}=F_{i} \cdot C_{i-1}^{i-1} \cdot F_{i}^{T}+Q_{i} \\
C_{i}^{i}=C_{i}-\frac{C_{i} \cdot H_{i}^{T} \cdot H_{i} \cdot C_{i}}{H_{i} \cdot C_{i} \cdot H_{i}^{T}+R_{i} / k_{i}} \\
k_{i}=\frac{\Psi\left(R_{i}^{-1 / 2} \cdot\left(X_{i}-H_{i} \cdot \hat{b}_{1}\right)\right)}{R_{i}^{-1 / 2} \cdot\left(X_{i}-H_{i} \cdot \hat{b}_{i}\right)} .
\end{gathered}
$$

Note that the 'ordinary' Kalman filter is just (3.4)-(3.6) with $k_{i}=1$.

For the special $\Psi=\Psi_{H}$ one gets from (3.2) in case $p=1$ the recursion:

$$
\hat{b}_{i}^{i}=\hat{b}_{i}+C_{i} \cdot H_{i}^{T} \cdot R_{i}^{-1 / 2} \cdot \Psi_{H}\left(\frac{R_{i}^{1 / 2} \cdot\left(X_{i}-H_{i} \cdot \hat{b}_{i}\right)}{H_{i} \cdot C_{i} \cdot H_{i}^{T}+R_{i}}\right)
$$

Cipra \& Romera propose to update $C_{l}$ like in the original (nonrobust) Kalmanfilter, i.e. :

$$
\begin{gathered}
C_{i}=F_{1} \cdot C_{i-1}^{i-1} \cdot F_{i}^{T}+Q_{i} \\
C_{i}^{i}=C_{1}-\frac{C_{i} \cdot H_{i}^{T} \cdot H_{i} \cdot C_{i}}{H_{i} \cdot C_{i} \cdot H_{i}^{T}+R_{i}} .
\end{gathered}
$$

Obviously the resulting recursions are quite tractable. 


\section{ROBUST CREDIBILITY}

We consider three well-known credibility models. For each model the robustifications (3.4)-(3.10) are presented, giving recursions for a robust credibility estimator.

\subsection{Bühlmann-Straub (1970) model}

Suppose that $X_{1}, \ldots, X_{n+1}$ are conditionally independent given $\theta$. There exist measurable functions

$$
\begin{aligned}
\mu:(\Theta, \tau) & \rightarrow(I R, I B) \\
\sigma^{2}:(\Theta, \tau) & \rightarrow\left(I R_{+}, I B_{+}\right)
\end{aligned}
$$

such that:

$$
\begin{gathered}
E\left(X_{i} \mid \theta\right)=\mu(\theta) \\
\operatorname{Var}\left(X_{i} \mid \theta\right)=\sigma^{2}(\theta) / V_{i},
\end{gathered}
$$

where $V_{i}, i \geq 1$ are known volume measures. Explicit formulas for the credibility estimator $\hat{m}_{n+1}$ can be found in the original article, equivalent recursions for it based on the classical Kalman filter in DE JONG et al. (1983). Obviously a dynamic linear model like in Section 3 is given with:

$$
\begin{array}{lll}
p=1, & H_{i}=1, & R_{i}=\sigma_{0}^{2} / V_{i} \\
q=1, & F_{1}=1, & Q_{i}=0,
\end{array}
$$

where:

$$
\sigma_{0}^{2}=E\left(\sigma^{2}(\theta)\right) .
$$

One has:

$$
\begin{aligned}
& b_{i}=m_{i}=\mu(\theta) \\
& \hat{b}_{i}=\hat{b}_{i-1}^{i-1}=\hat{m}_{i}, \quad C_{i}=C_{i-1}^{i-1}
\end{aligned}
$$

implying from (3.4), (3.6), (3.7) in case of general $\psi$ :

$$
\begin{gathered}
\hat{m}_{i+1}=\hat{m}_{i}+\left(\frac{C_{i} \cdot V_{i} \cdot k_{i}}{C_{i} \cdot V_{i} \cdot k_{i}+\sigma_{0}^{2}}\right) \cdot\left(X_{i}-\hat{m}_{i}\right) \\
C_{i+1}=C_{i}-\frac{C_{i}^{2} \cdot V_{i} \cdot k_{1}}{C_{i} \cdot V_{i} \cdot k_{i}+\sigma_{0}^{2}} \\
k_{i}=\frac{\Psi\left(V_{i}^{1 / 2} \cdot\left(X_{i}-m_{1}\right) / \sigma_{0}\right)}{V_{i}^{1 / 2} \cdot\left(X_{i}-m_{i}\right) / \sigma_{0}}
\end{gathered}
$$


and from (3.8), (3.10) in case of special $\Psi=\Psi_{H}$ :

$$
\begin{gathered}
\hat{m}_{i+1}=\hat{m}_{i}+C_{i} \cdot\left(\frac{V_{i}^{1 / 2}}{\sigma_{0}}\right) \cdot \Psi_{H}\left(\frac{\sigma_{0} \cdot V_{i}^{1 / 2} \cdot\left(X_{i}-\hat{m}_{i}\right)}{C_{i} \cdot V_{i}+\sigma_{0}^{2}}\right) \\
C_{i+1}=\left(V_{i} / \sigma_{0}^{2}+C_{1}^{-1}\right)^{-1} .
\end{gathered}
$$

Obviously one has quite handy recursions for the (robust) credibility estimator $\hat{m}_{i}$.

\subsection{Hachemeister's (1975) regression model}

Conditionally given $\theta$ the $X_{1}, \ldots, X_{n+1}$ are independent. Suppose that there exist functions:

$$
\begin{aligned}
& b:(\Theta, \tau) \rightarrow\left(I R^{q}, I B^{q}\right) \\
& \sigma^{2}:(\Theta, \tau) \rightarrow\left(I R_{+}, I B_{+}\right)
\end{aligned}
$$

such that:

$$
\begin{gathered}
E\left(X_{i} \mid \theta\right)=a_{i}^{T} \cdot b(\theta) \\
\operatorname{Var}\left(X_{i} \mid \theta\right)=\sigma^{2}(\theta) / V_{i},
\end{gathered}
$$

where $a_{i}$ is a known $q$-dimensional vector and $V_{i}, i \geq 1$ are given volume measures. The credibility estimator $\hat{m}_{n+1}$ of $m_{n+1}$ is given by:

$$
\hat{m}_{n+1}=a_{n+1}^{T} \cdot \hat{b}_{n+1},
$$

where $\hat{b}_{n+1}$ is the (vector-valued) credibility estimator of $b(\theta)$ based on $X_{1}, \ldots, X_{n}$.

Explicit formulas for $\hat{b}_{n+1}$ can be found in the original paper of HACHEMEISTER (1975), equivalent recursions based on the classical Kalman-filter in DE JONG \& ZEHNWIRTH (1983).

Obviously the model fits into the framework of Section 3.

Choose simply there:

$$
\begin{array}{ll}
p=1, & H_{i}=a_{i}^{T}, \quad R_{i}=\sigma_{0}^{2} / V_{i} \\
F_{i}=1, & Q_{i}=1,
\end{array}
$$

where :

$$
\sigma_{0}^{2}=E\left(\sigma^{2}(\theta)\right)
$$

One has:

$$
\begin{aligned}
& b_{i}=b(\theta) \\
& b_{i}=b_{i-1}^{i-1}, \quad C_{i}=C_{i-1}^{i-1} .
\end{aligned}
$$


The recursion (3.4), (3.6), (3.7) give in case of general $\Psi$ :

$$
\begin{gathered}
\hat{b}_{i+1}=\hat{b}_{i}+\left(\frac{C_{i} \cdot a_{i} \cdot V_{i} \cdot k_{i}}{a_{i}^{T} \cdot C_{i} \cdot a_{i} \cdot V_{i} \cdot k_{i}+\sigma_{0}^{2}}\right) \cdot\left(X_{i}-a_{i}^{T} \cdot \hat{b}_{i}\right) \\
C_{i+1}=C_{i}-\left(\frac{C_{i} \cdot a_{i} \cdot a_{i}^{T} \cdot C_{i} \cdot V_{i} \cdot k_{i}}{a_{i}^{T} \cdot C_{i} \cdot a_{i} \cdot V_{i} \cdot k_{i}+\sigma_{0}^{2}}\right) \\
k_{i}=\left(\frac{\Psi\left(V_{i}^{1 / 2} \cdot\left(X_{i}-a_{i}^{T} \cdot \hat{b}_{i}\right) / \sigma_{0}\right)}{V_{i}^{1 / 2} \cdot\left(X_{i}-a_{i}^{T} \cdot \hat{b}_{i}\right) / \sigma_{0}}\right)
\end{gathered}
$$

and the recursions (3.8)-(3.10) in case of special $\Psi=\Psi_{H}$ :

$$
\begin{aligned}
& \hat{b}_{i+1}=\hat{b}_{i}+C_{i} \cdot a_{1} \cdot\left(\frac{V_{i}^{1 / 2}}{\sigma_{0}}\right) \cdot \Psi_{H}\left(\frac{\sigma_{0} \cdot V_{i}^{1 / 2} \cdot\left(X_{i}-a_{i}^{T} \cdot \hat{b}_{i}\right)}{a_{i}^{T} \cdot C_{i} \cdot a_{i} \cdot V_{1}+\sigma_{0}^{2}}\right) \\
& C_{i+1}=C_{i}-\left(\frac{C_{i} \cdot a_{i} \cdot a_{i}^{T} \cdot C_{i} \cdot V_{1}}{a_{i}^{T} \cdot C_{i} \cdot a_{i} \cdot V_{i}+\sigma_{0}^{2}}\right) .
\end{aligned}
$$

Obviously also these recursions for a (robust) credibility estimator are quite practicable.

\subsection{Gerber \& Jones' (1975) evolutionary model}

Suppose that $X_{1}, \ldots, X_{n+1}$ are conditionally independent given $\theta$. Furthermore assume that:

$$
m_{i}=m_{i-1}+w_{i},
$$

where the random disturbances satisfy:

$$
\begin{gathered}
E\left(w_{i}\right)=0, \quad E\left(w_{i}^{2}\right)=w \\
E\left(w_{i} \cdot w_{j}\right)=0, \quad i \neq j, \quad E\left(w_{i} \cdot m_{0}\right)=0 .
\end{gathered}
$$

Finally let :

$$
\sigma^{2}:(\Theta, \tau) \rightarrow\left(I R_{+}, I B_{+}\right)
$$

Such that:

$$
\operatorname{Var}\left(X_{i} \mid \theta\right)=\sigma^{2}(\theta) / V_{i},
$$

where $V_{i}, i \geq 1$ are given volume measures. Recursions for the credibility estimator are given e.g. in the paper SUNDT (1981).

This model is a special case of the dynamic linear model of Section 3 . Choose simply there:

$$
\begin{array}{ll}
p=1, & H_{i}=1, \quad R_{i}=\sigma_{0}^{2} / V_{i} \\
F_{i}=1, & Q_{i}=w,
\end{array}
$$

with :

$$
\sigma_{0}^{2}=E\left(\sigma^{2}(\theta)\right)
$$


The recursions (3.4), (3.6), (3.7) give in case of general $\Psi$ :

$$
\begin{array}{r}
\hat{m}_{i+1}=\hat{m}_{i}+\left(\frac{C_{i} \cdot V_{i} \cdot k_{i}}{C_{i} \cdot V_{t} \cdot k_{i}+\sigma_{0}^{2}}\right) \cdot\left(X_{i}-\hat{m}_{i}\right) \\
C_{i+1}=C_{i}-\frac{C_{i}^{2} \cdot V_{i} \cdot k_{i}}{C_{i} \cdot V_{i} \cdot k_{i}+\sigma_{0}^{2}}+w \\
k_{i}=\frac{\Psi\left(V_{i}^{1 / 2} \cdot\left(X_{i}-\hat{m}_{t}\right) / \sigma_{0}\right)}{V_{i}^{1 / 2} \cdot\left(X_{i}-\hat{m}_{i}\right) / \sigma_{0}}
\end{array}
$$

and the recursions (3.8), (3.10) in case of special $\psi=\Psi_{H}$ :

$$
\begin{gathered}
\hat{m}_{i+1}=\hat{m}_{i}+\left(C_{i}+w\right) \cdot\left(\frac{V_{i}^{1 / 2}}{\sigma_{0}}\right) \cdot \Psi_{H}\left(\frac{\sigma_{0} \cdot V_{i}^{1 / 2} \cdot\left(X_{i}-\hat{m}_{i}\right)}{C_{i} \cdot V_{i}+w \cdot V_{i}+\sigma_{0}^{2}}\right) \\
C_{i+1}=C_{i}-\frac{C_{i}^{2} \cdot V_{i}}{C_{i} \cdot V_{i}+\sigma_{0}^{2}}+w .
\end{gathered}
$$

\section{A SIMULATION STUDY}

For the model of Section 4.1 data was simulated with the choice $V_{i}=1$ for all $i$. The conditional distribution of $X_{i}$ given $\theta=\vartheta$ was assumed to be given according

$$
\begin{gathered}
P\left(X_{i}=k \mid \theta=\vartheta\right)=(1-\pi) \cdot p_{\vartheta}(k)+\pi \cdot p_{\vartheta_{0}}(k) \\
\text { (for } k=0,1,2,3, \ldots)
\end{gathered}
$$

with the Poisson-probabilities:

$$
p_{\vartheta}(k)=\left(\frac{\vartheta^{k}}{k !}\right) \cdot \exp (-\vartheta)
$$

and a probability $\pi$. For $\vartheta_{0}>\vartheta \pi$ can be interpreted as the probability of an outlier occurring according to the probabilities $p_{\vartheta_{0}}(k)$. The risk parameter $\vartheta$ was simulated according to the Gamma-model with density on $(0, \infty)$ :

$$
f^{\vartheta}(\vartheta)=\frac{\beta^{\alpha}}{\Gamma(\alpha)} \cdot \vartheta^{\alpha-1} \cdot \exp (-\beta \cdot \vartheta)
$$

where $\alpha$ and $\beta$ are the nonnegative parameters. One gets for $\pi=0$ as credibility estimator:

$$
\hat{m}_{n+1}=\frac{\alpha+\sum_{i=1}^{n} X_{i}}{\beta+n}
$$


what shall be compared with a robust variant calculated recursively according to the formulas :

$$
\begin{gathered}
\hat{m}_{n+1}=\hat{m}_{n}+\left(C_{n} / \sigma_{0}\right) \cdot \Psi_{H} \cdot\left(\frac{X_{n}-\hat{m}_{n}}{\left(C_{n} / \sigma_{0}\right)+\sigma_{0}}\right) \\
C_{n+1}=\frac{\sigma_{0}^{2}}{1+\left(\sigma_{0}^{2} / C_{n}\right)}
\end{gathered}
$$

where $\sigma_{0}^{2}=(\alpha / \beta)$ and $\Psi_{H}$ is the Huber-function with $c=1.645$. The recursions start with:

$$
\hat{m}_{1}=(\alpha / \beta), \quad C_{1}=\left(\alpha / \beta^{2}\right) .
$$

The aim of the study was to compare the results of (5.3), (5.4) with the 'true' value $\vartheta$ and to see which one gives the smaller mean squared error:

$$
\frac{1}{n-n_{0}+1} \cdot \sum_{i=n_{0}}^{n}\left(\hat{m}_{i+1}-\vartheta\right)^{2},
$$

where $n_{0}$ is an adequate number smaller than $n$. The claims data had to be simulated with a sufficiently large $\vartheta_{0}$ and an adequate small $\pi$. The author chosed $\pi=0.05$ and for $\vartheta_{0}$ the values 20,25,30, whereas he took $\alpha=100, \beta=10$, giving for $E(\theta)$ the value 10 . With these parameter choices he simulated 100 risk parameters $\vartheta_{\text {, }}$, $j=1, \ldots, 100$ according to the model (5.2) and for each $\vartheta=\vartheta_{j}$ independently $n=9$ values $X_{i}$ according to (5.1) (with $\pi=0.05$ ). In (5.5) he took $n_{0}=6$. He got for the overall mean squared error

$$
M S E=\frac{1}{500} \cdot \sum_{j=1}^{100} \sum_{i=6}^{10}\left(\hat{m}_{i+1}^{(j)}-\vartheta_{j}\right)^{2}
$$

the results of the following table:

\begin{tabular}{l|ccc}
\hline \hline & $\vartheta_{0}=20$ & 25 & 30 \\
\hline with & & & \\
$(5.3)$ & 0.956 & 1.231 & 1.593 \\
$(5.4)$ & 0.806 & 0.807 & 0.807 \\
\hline
\end{tabular}

showing the strong superiority of (5.4) for situations where bigger outliers can occur with small probability but one wants to rate the normal risk (i.e. case $\pi=0$ ). For further illustration the simulation results shall be given for two typical cases. In the first row of the following tables the simulated $X_{i}$ are given, in the second the $\hat{m}_{i}$ of (5.3) and in the third the $\hat{m}_{1}$ of (5.4).

$\vartheta_{0}=\mathbf{2 0}$ : 
$\vartheta_{j}=11.30$

\begin{tabular}{cccccccccc}
\hline \hline 9 & 13 & 11 & 22 & 13 & 15 & 14 & 14 & 16 & - \\
\hline 10.00 & 9.91 & 10.17 & 10.23 & 11.07 & 11.20 & 11.44 & 11.59 & 11.72 & 11.95 \\
\hline 10.00 & 9.91 & 10.17 & 10.23 & 10.63 & 10.79 & 11.05 & 11.23 & 11.38 & 11.62 \\
\hline
\end{tabular}

$\vartheta_{j}=8.42$

\begin{tabular}{cccccccccc}
\hline \hline 21 & 8 & 12 & 9 & 4 & 8 & 9 & 19 & 8 & - \\
\hline 10.00 & 11.00 & 10.75 & 10.85 & 10.71 & 10.27 & 10.13 & 10.06 & 10.56 & 10.42 \\
\hline 10.00 & 10.52 & 10.31 & 10.44 & 10.34 & 9.91 & 9.80 & 9.75 & 10.05 & 9.95 \\
\hline
\end{tabular}

$\vartheta_{0}=25$

$\vartheta_{j}=9.60$

\begin{tabular}{rccccccccc}
\hline \hline 7 & 19 & 11 & 11 & 11 & 33 & 12 & 11 & 11 & - \\
\hline 10.00 & 9.73 & 10.50 & 10.54 & 10.57 & 10.60 & 12.00 & 12.00 & 11.94 & 11.89 \\
\hline 10.00 & 9.73 & 10.20 & 10.26 & 10.31 & 10.36 & 10.70 & 10.78 & 10.80 & 10.81 \\
\hline
\end{tabular}

$\vartheta_{j}=10.71$

\begin{tabular}{ccccccccccc}
\hline \hline 12 & 8 & 24 & 12 & 15 & 15 & 10 & 13 & 11 & - \\
\hline 10.00 & 10.18 & 10.00 & 11.08 & 11.14 & 11.40 & 11.63 & 11.53 & 11.61 & 11.58 \\
\hline 10.00 & 10.18 & 10.00 & 10.43 & 10.55 & 10.84 & 11.10 & 11.03 & 11.15 & 11.14 \\
\hline
\end{tabular}

$\boldsymbol{\vartheta}_{0}=\mathbf{3 0}$ :

$\vartheta_{j}=10.77$

\begin{tabular}{cccccccccc}
\hline \hline 11 & 7 & 13 & 6 & 7 & 28 & 7 & 40 & 11 & - \\
\hline 10.00 & 10.09 & 9.83 & 9.79 & 9.60 & 9.60 & 10.75 & 10.53 & 12.17 & 12.11 \\
\hline 10.00 & 10.09 & 9.83 & 10.08 & 9.79 & 9.60 & 9.95 & 9.77 & 10.08 & 10.13 \\
\hline
\end{tabular}


$\vartheta_{j}=8.42$

\begin{tabular}{cccccccccc}
\hline \hline 31 & 8 & 12 & 9 & 4 & 8 & 9 & 29 & 8 & - \\
\hline 10.00 & 11.91 & 11.58 & 11.62 & 11.43 & 10.93 & 10.75 & 10.65 & 11.67 & 11.47 \\
\hline 10.00 & 10.52 & 10.31 & 10.44 & 10.34 & 9.91 & 9.80 & 9.75 & 10.05 & 9.95 \\
\hline
\end{tabular}

\section{FINAL REMARKS}

By applying robustifications of the Kalman-filter to credibility models one can derive fairly practicable recursions for a (robust) credibility estimator. For the Bühlmann-Straub and Hachemeister models one gets an alternative to an already existing approach to robust credibility (see KREMER (1991), KüNSCH (1992)). For practical application of the above robustified recursions one needs (robust) estimators for the unknown model parameters. Desirable would be such estimators in a recursive form. Obviously here is something left for further research.

\section{REFERENCES}

BUHL.MANN, H. (1967) Experience rating and credibility. ASTIN Bullerin.

BÚhlmanN. H. and STRAUB, E. (1970) Glaubwürdigkeit für Schadensätze. Mitteilungen der Vereinigung Schweizerischer Versicherungsmathemasiker.

Buhi_mann, H., Gisl_er. A. and Jewell, W.S. (1982) Excess claims and data trimuning in the context if credibility rating procedures. Mitreilungen der Vereinigung Schweizerischer Versicherungsmathematiker.

Cipra, T. and Romera, R. (1991) Robust Kalman filter and its application in time series analysis. Kubernesika.

De Jong, P. and Zehnwirth, B. (1983) Credibility theory and the Kalman filter. Insurance : Mathemarics and Economics.

Gerber, H.U. and Jones, D.A. (1975) Credibility formulas of the updating type. Transactions of the society of actuaries.

GisLER, A. (1980) Optimum trimming of data in the credibility model. Mitteilungen der Vereinigumg Schweizerischer Versicherungsmathematiker.

Gisler, A. and ReinhaRd, P. (1993) Robust Credibility. ASTIN Bulletin.

HACHEMEISTER, Ch. (1975) Credibility for regression models with application to trend. In Credibility: Theory and applications (ed. P. M. KAHN). New York.

Kremer, E. (1988a) Box-Jenkins Credibility. Blätter der deutschen Gesellschat fiir Versicherungsmathematiker.

KREMER, E. (1988b): General exponential smoothing credibility. Bulletin of the Belgian Acruarial Association.

KREMER, E. (1991) Large claims in credibility. Blätter der deutschen Gesellschaft für Versicherungsmathemasik.

KUnNSCH, H.R. (1992) Robust methods for credibility. ASTIN Bulletin.

MASREliez, C.J. and MARTIN, R.D. (1977) Robust Bayesian estimation for the linear model and robustifying the Kalman filter. IEEE Transactions on Automaric Control AC 22.

MehrA, R.K. (1975) Credibility theory and Kalman filtering with extensions. Imernational Instituse for applied system analysis. Laxenburg, Austria.

Meinhold, R. J. and Singpurwal._A, N.D. (1989) Robustification of Kalman filter models. Journal of the American Statistical Association 84. 
Mowbray, A.H. (1914) How extensive a payroll exposure is necessary to give a dependable pure premium. Proceedings of the Casualy Actuarial society.

Norberg, R. (1980) Empirical Bayes credibility. Scandinavian Actuarial Journal.

NORBERG, R. (1986) Hierarchical credibility: Analysis of a random effect linear model with nested classification. Scandinavian Actuarial Journal.

SUNDT. B. (1980) A multi-level hierarchical credibility model. Scandinavian Acruarial Journal.

SundT, B. (1981) Recursive credibility estimation. Scandinavian Actuarial Journal.

Sundr. B. (1983) Finite credibility formulae in evolutionary models. Scandinavian Actuarial Journal.

TAYI_OR G.C. (1979) Credibility analysis of a general hierarchical model. Scandinavian Actuarial Journal.

Whitney, A.W. (1918) The theory of experience rating. Proceedings of the Casualty Actuarial society:

ZEHNWIRTH, B. (1975) A linear filtering approach to recursive credibility estimation. ASTIN Bulletin.

\section{ERHARD KREMER \\ Institut für Mathematische Stochastik, Bundesstraße 55, 20146 Hamburg, FRG.}


